$\begin{array}{ll} & \text { Etnográfica } \\ \text { etnográfica } & \text { Revista do Centro em Rede de Investigação em }\end{array}$

Antropologia

vol. 18 (1) | 2014

Vol. $18(1)$

\title{
Des hommes en fêtes et en armes : analogies guerrières et formes d'atténuation
}

Homens em festa e em armas : analogias guerreiras e formas de atenuação

\section{Danièle Dossetto}

\section{(2) OpenEdition}

\section{Journals}

Édition électronique

URL : https://journals.openedition.org/etnografica/3341

DOI : 10.4000/etnografica.3341

ISSN : 2182-2891

\section{Éditeur}

Centro em Rede de Investigação em Antropologia

\section{Édition imprimée}

Date de publication : 1 février 2014

Pagination : 53-76

ISSN : 0873-6561

\section{Référence électronique}

Danièle Dossetto, «Des hommes en fêtes et en armes : analogies querrières et formes d'atténuation », Etnográfica [En ligne], vol. 18 (1) | 2014, mis en ligne le 14 mars 2014, consulté le 11 février 2022. URL : http://journals.openedition.org/etnografica/3341 ; DOI : https://doi.org/10.4000/etnografica.3341

\section{(c) (7) \&}

Etnográfica is licensed under a Creative Commons Attribution-NonCommercial 4.0 International License. 


\section{Des hommes en fêtes et en armes : analogies guerrières et formes d'atténuation}

\section{Danièle Dossetto}

Les fêtes armées, sans adopter forcément l'allure de batailles, font usage de tirs à blanc; tandis que, dans la péninsule ibérique et dans le nouveau monde, ce sont les fêtes de Maures et chrétiens, en Provence-Alpes-Côte d'Azur, ce sont les "bravades". En procédant à quelques rapprochements entre ces deux formules localisées, le propos est d'apporter des éléments de réflexion sur les conditions de représentation de la guerre. Quels liens ces fêtes entretiennent-elles avec de réels et plus ou moins récents conflits armés? Quels usages métaphoriques peut-il leur être donné? Comment surtout mettre en œuvre des armes à feu dans une fête aujourd'hui? Le burlesque carnavalesque, des attitudes paradoxales chez les hommes en armes, l'exploitation d'un certain parallélisme avec la chasse, le vêtement, le recours à la candeur enfantine... sont autant de formules attestées ici ou là pour aménager ce qui apparaît comme un fait de tradition en porte-à-faux.

MOTS-CLEFS : "bravade " et fêtes armées, poudre festive, armes à feu, genre et âge, tradition, Provence-Alpes-Côte d'Azur.

Homens em festa e em armas: analogias guerreiras e formas de atenuação - As festas armadas, sem adotar forçosamente o aspeto de batalhas, recorrem a tiros de pólvora seca; na Península Ibérica e no novo mundo há as festas de mouros e cristãos e, na região de Provence-Alpes-Côte d'Azur, as bravades. Graças a algumas aproximações entre estas duas fórmulas localizadas, propõem-se elementos de reflexão acerca das condições de representação da guerra. Que elos existem entre estas festas e conflitos armados reais e mais ou menos recentes? Que usos metafóricos podem ter? Como, sobretudo, recorrer a armas de fogo numa festa hoje? O burlesco carnavalesco, algumas atitudes paradoxais dos homens em armas, a exploração de um certo paralelismo com a caça, o traje, o recurso à candura infantil, constituem fórmulas, testemunhadas em ambos os contextos, destinadas a ajustar o que parece ser um facto de tradição em equilíbrio instável.

PALAVRAS-CHAVE: bravade e festas armadas, pólvora festiva, armas de fogo, género e idade, tradição, Provence-Alpes-Côte d'Azur.

DOSSETTO, Danièle (daniele.dossetto@unice.fr) - Laboratoire d'Anthropologie et de Psychologie Cognitives et Sociales (LASMIC/LAPCOS), Université de NiceSophia Antipolis, France. 
NOMBREUSES SONT LES RÉGIONS À S'ILLUSTRER PAR DES FÊTES ARMÉES. Un colloque récent a étudié des fêtes de Maures et chrétiens, Morescas, Morismas ou Soldatescas en Espagne, au Portugal ou en Italie (Albert-Llorca et González-Alcantud 2003); on sait par ailleurs que, d'Europe, elles se sont exportées en Amérique du sud (Carrasco 1976). Entre péninsule ibérique et Italie, la Provence-Alpes-Côte d'Azur connaît aussi la pratique festive des armes, cela sous le terme de "bravade". D'autres exemples sont les "alardes" du pays Basque ou les "marches" de l'Entre-Sambre-et-Meuse belge. La grande extension des fêtes armées et leur vivacité actuelle appellent une question: comment peut-on aujourd'hui exhiber des armes, a fortiori figurer la guerre, pour en faire une pratique festive? Concrètement, l'arme à feu fondant la "bravade" qui va nous occuper, ${ }^{1}$ notre question initiale peut se reformuler ainsi: a quelles manipulations le syndrome "fusil" en fête donne-t-il lieu? Comment des acteurs décideurs accommodent-ils des réjouissances aux allures guerrières? Quels exemples a-t-on de compromis avec ce fait de tradition?

La matière disponible est encore limitée sur ces divers points. En ce qui concerne les fêtes de Maures et chrétiens, en tout cas pour les lecteurs français, la région d'Alicante est la plus connue sous l'angle ethnologique (Albert-Llorca 1995, 2004; Albert-Llorca et Albert 1995; Albert-Llorca et Blanc 2001 ) ; c'est là que je trouverai des observations utiles. ${ }^{2}$ Pour les “bravades", peu de chercheurs se sont intéressés à leurs modalités présentes; je recourrai donc surtout à des enquêtes personnelles dans le Var. Une telle comparaison est favorisée par certaines données. La forte adhésion des habitants est un point commun entre la Provence varoise et la province d'Alicante. A Saint-Tropez - qui fournit la "bravade" la plus célèbre -, ceux qui manipulent la poudre représentent 3,2\% de la population et les 250 "bravadeurs" (ici au sens d'hommes en armes ou musiciens en uniforme), 4,5\% ; cette dernière proportion est en augmentation depuis un demi-siècle, où elle était de 3,4\%; 7\% des résidents de Villajoyosa sont festers "Maures" ou "chrétiens". Mais le nombre des membres de l'association soutenant la Bravade correspond à $27 \%$ de la population tropézienne sans réunir pour autant tous les fidèles de la fête; qu'un tiers des habitants soit impliqué, voilà qui est exactement la proportion relevée à Villena. Autres signes de ferveur, dans les deux régions, la participation à une fête armée exige une contribution financière individuelle parfois lourde (Albert-Llorca 1995 ; Buyret 1954). Un intérêt local massif rejoint ainsi les affinités formelles pour justifier

l Tout en y intervenant quelquefois, la hallebarde et l'épée ont en effet des aires de diffusion qui en sont indépendantes. La première n'a une visibilité réelle que dans les Alpes-Maritimes (Canestrier 1955), la seconde (Carenini 1996) dans les Hautes-Alpes; l'image de la danse armée qui est cultivée dans ce dernier cas orienterait d'ailleurs les comparaisons vers l'Europe de l'est (voir, par exemple, Capo-Zmegac 2004) plus que vers l'Espagne, du côté de laquelle nous aurons à nous tourner.

2 J'ai en outre procédé au dépouillement de la Revista de dialectología y tradiciones populares (série lacunaire conservée à Aix-en-Provence, Maison méditerranéenne de sciences de l'homme). 
un parallèle. D'autres investigations nourriraient le dossier; tel quel pourtant, il est assez consistant pour être proposé à l'attention collective; le lecteur voudra bien voir dans ces pages les prémices d'un défrichage et le repérage d'une direction interprétative qui pourrait être éprouvée sur d'autres terrains. ${ }^{3}$

\section{UNE PRATIQUE EN EXPANSION}

A l'échelle de la Provence-Alpes-Côte d'Azur, le phénomène "bravade" se présente comme le legs de milices assurant la sécurité des lieux, mais aussi, les coups de feu étant associés à des processions, comme celui d'un clergé soucieux, au XVII siècle, de ranimer la ferveur de ses ouailles; une carte pour les années 1780 (Vovelle 1976: 43) montre combien la pratique a été répandue. Souvent, l'emploi des armes est articulé à un récit étiologique - légendaire religieux ou autre -, dont la formation tardive et tâtonnante a par exemple été mise au jour pour Saint-Tropez et Fréjus (Var) (Février 1966). Il est évidemment des manifestations de type "bravade" en toute saison, mais cet usage, qui s'est mieux maintenu dans la région orientale - Var, Alpes-de-Haute-Provence, AlpesMaritimes -, entretient un rapport étroit avec la variante "cavalcade" de la Saint-Eloi d'été, fête équine aux antécédents agricoles (Dossetto 2004a). Parmi mes exemples varois, Saint-Tropez représentera une "bravade" aux allures militaires, Signes une Saint-Eloi et Barjols une fête patronale où la mitraille, assez importante d'un point de vue objectif, perd de l'importance par rapport à d'autres descripteurs. ${ }^{4}$ On l'aura compris, selon les cas, le terme de "bravade" désigne soit la fête soit une séquence ou un élément de celle-ci; de même sont "bravadeurs" (ou "bravadiers", 5 selon les localités) les seuls hommes en armes (cas dominant) ou tous les figurants (comme à Saint-Tropez).

Pour ce qui est de la période qui nous est contemporaine, après quelques décennies d'hésitation, le genre "bravade" a connu un important regain. ${ }^{6}$

3 Le présent article reprend une conférence donnée au Musée de l'artillerie (Draguignan, Var) en octobre 2006. Je remercie Hassan Moukhlisse (Maison méditerranéenne des sciences de l'homme) pour la numérisation des clichés. La luminosité du rendu est à peu près conforme à la réalité pour les photos $4 \mathrm{a}$ et $4 \mathrm{~b}$ (belles journées de janvier), mais elle la transforme à Saint-Tropez, où il faut considérer que les clichés ont été réalisés au cours de journées quasi estivales; les vues des séquences de tirs doivent être appréciées à partir de l'assombrissement général de la série.

4 Par exemple un bœuf auquel il me faudra faire allusion plus tard; la densité des tirs est moindre quand l'animal est présent (un an sur trois aujourd'hui); alors que la fête prend généralement plus d'éclat (chevaux etc.), la nomination d'un "capitaine de ville" (infra) n'intervient que ces années-là, mais c'est bien le bœuf qui attire prioritairement les visiteurs à Barjols.

5 Le terme provençal est "bravadaire" ("bravade" se dit "bravado").

6 Une analyse des phénomènes généraux de réactivation (revitalization) qui en constituent le cadre est accessible dans Dossetto (2004b), contribution à un colloque de 1999 sur cette thématique; pour ce qui concerne le fait "bravade" seul, on se reportera plutôt à Dossetto (2009). Cela dit, les faits que nous aurons à examiner ne coïncident pas nécessairement avec des phénomènes de réactivation. 
Dans les Alpes-de-Haute-Provence, par exemple, des années 1940 ou 1950, à 1978, la désuétude du phénomène s'est renversée de telle sorte qu'à cette dernière date une enquête a mis au jour une demi-douzaine de manifestations armées : à Gréoux ou à Annot (dans ce département), des restaurations ont eu lieu en 1953 ou 1958-1960 (puis 1973). Cependant de telles réactivations ne sont pas concentrées dans l'arrière région; sur la côte varoise, par exemple, les "bravades" de Fréjus, Sainte-Maxime, Cogolin ou Lagarde-Freinet ont repris en 1952, 1968, 1969 et 1983.

Un autre indicateur de dynamisme est le spectaculaire développement de "bravades" établies. A Saint-Tropez par exemple, au milieu des années 1950 c'est-à-dire plus de trente ans après une réactivation réussie et déjà sur une courbe ascendante, on décompte une centaine de fusils et tromblons et 20-25.000 tirs en trois jours, soit 200-250 kg de poudre. Aujourd'hui, avec presque 180 armes et à raison de 2 à $4 \mathrm{~kg}$ de consommation moyenne selon les catégories d'hommes en armes ("marins", "mousquetaires", "garde-saint"), on parvient au total de 400 à $500 \mathrm{~kg}$ de poudre pour environ 220 "saluts " (tirs groupés devant une personne honorée ou son succédané, comme une statue). ${ }^{7}$ Entre nos deux repères temporels, l'accroissement concerne donc aussi bien le nombre d'hommes en armes que la consommation individuelle moyenne de poudre.

De plus, il faut noter l'élasticité actuelle des pratiques. La valorisation comme "provençal" du fait "bravade" fonde des phénomènes de diffusion ou d'intégration dans des fêtes nouvelles. A Salon-de-Provence (Bouches-du-Rhône), dans une fête de la transhumance qui, comme ses homologues, est une innovation du XXe siècle finissant, un "bravadeur" intervient aux côtés du troupeau qui rappelle les départs pédestres vers les pâturages alpins. A Aix-en-Provence (Bouches-du-Rhône), une Bravade avec offrande aux élus intègre désormais les festivités de fin d'année. En même temps, la prise d'importance des fêtes armées dans le blason local produit des physionomies caractérisées de manière croissante. Par exemple, par le choix des armes, le répertoire musical ou le vêtement, les "bravades" de Saint-Tropez, de Barjols et d'Aix se sont personnalisées sur la base de modèles formels et d'individus circulant de l'une à l'autre.

Une telle effervescence ouvre largement l'éventail des situations.

\section{DES “MUSULMANS”: ENNEMIS OU FIGURES DE SOI?}

Commençons l'examen par ce qui assure le lien le plus net avec les fêtes ibériques, à savoir les cas prenant l'allure de confrontations interreligieuses ou

7 A Barjols, on estime qu'il y a 150 à 250 cartouches par homme, sur la base de 22 grammes de poudre par cartouche et dix armes (appartenant à l'association organisatrice). A Signes, chaque homme disposerait d'à peu près 200 cartouches. C'est donc davantage le nombre de tireurs que la consommation de chacun qui différencie nos exemples. 
procédant au traitement festif d'une caractérisation musulmane ; leur rareté en Provence-Alpes-Côte d'Azur nous oblige à une incursion dans le passé.

Essentiel dans les fêtes de Maures et chrétiens, l'assaut d'un château a ici complètement disparu. Quelques équivalents se concentraient dans les Alpesde-Haute-Provence actuelles, ${ }^{8}$ où le mieux documenté est celui de Riez. Les attaques d'un fort provisoire y ont énormément fluctué, avec toutefois pour constante de ne pas inverser la position des deux camps, l'occupation alternative du bastion semblant au contraire une règle en Espagne. En 1808, un voyageur "antiquaire" définit ce "guet" (sic) se déployant sur plusieurs jours comme " une bravade entre les Chrétiens et les Sarrasins". Le bipartisme religieux est alors assuré. Il est en revanche inconnu d'un témoin ayant pratiqué la fête quelques décennies après et consignant ses souvenirs encore un peu plus tard, en 1907, après une disparition définitive. A l'en croire, la "bravade" des derniers temps se réduisait à une exhibition intéressant peu le public, un spectacle au temps mesuré et sans enjeux manifestes. Attaques raccourcies (un jour), fond musical, déroulement du combat en même temps qu'une foire affaiblissaient encore l'analogie guerrière. Alors qu'il se consommait tout de même "des quintaux de poudre", les ennemis festifs n'avaient plus de caractère net bien que le combat pût sans doute évoquer les grandes batailles napoléoniennes puisque "le nom d'une grande victoire de nos armées" figurait au fronton du "fort" et que l'on utilisait toutes les "défroques militaires" disponibles. Le thème d'un conflit religieux était en tout cas oublié et la disparition de la fête à la fin du XIX siècle sanctionne peut-être un déficit de sens.

A défaut d'échauffourées autour d'une citadelle, l'actualité festive simule toujours des "Sarrasins" ou "Turcs" dans la Stacada de Breil (Alpes-Maritimes). La forme de conflit que prend cette fête (Lebaudy 1998) suggère d'ailleurs une évolution intéressante pour notre propos. En 1914, d'après une affiche, l' "estaquade" "représente la révolte des 'serfs" contre les Seigneurs du pays"; en 1947, dernier sursaut avant une interruption, sont créés les personnages d'un "seigneur" et d'une "mariée", en vérité un homme travesti car nous sommes en période de Carnaval; en 1960, date de réactivation en juillet, intervient une menace de droit de cuissage sur la "mariée" et, au cours de la révolte populaire qui s'ensuit, le "bailli" qui a prétendu en user encourt la décapitation. Le durcissement des "seigneurs" donne un motif plausible à une émeute sociale. Les "Turcs" y participent au côté de la plèbe si bien que la raison de leur exotisme n'apparaît pas. Cela donne à penser à un rôle douteux - changeant - sur la durée : les "Turcs", par le passé, ont peut-être été la cible des "dragons" (soldats) qui interviennent dans la fête. Moyennant probablement une évolution des confrontations et des alliances entre groupes singularisés, ce qui est significatif pour nous est que la Stacada, in fine, évite la 
représentation d'un conflit religieux. Elle résorbe en outre la tension qui existe entre respect d'une tradition guerrière et pacifisme ambiant en immergeant l'usage des armes dans un complexe festif bon enfant (permanence d'éléments carnavalesques malgré une célébration désormais estivale) et en en fournissant une interprétation moderniste, des "notables" ayant succédé aux "seigneurs" comme contraires aux intérêts du "peuple".

Pauvre en combats festifs, dotée de "bravades" moins que jamais rapportées à une irréductible altérité ethnico-religieuse sarrasine, la Provence-AlpesCôte d'Azur offre en contrepartie aux représentations un terreau cultuel plus varié que la région d'Alicante puisque le schéma de l'appartenance religieuse ou confessionnelle figurée y est (ou y a été) tripolaire. La référence au protestantisme singularise en effet certaines "bravades". Dans le Var, celle de Barjols, selon une plume du XIXe siècle, rappelle les "guerres de religion" auxquelles le village a fourni un cadre avéré. Dans les Alpes-de-Haute-Provence, à Castellane, l'étiologie confessionnelle s'est maintenue dans la commémora-

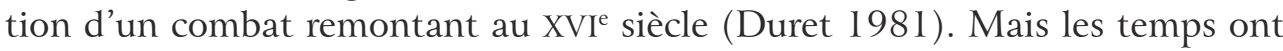
changé. A Barjols, le thème narratif ancien s'est oublié et les organisateurs actuels ne confèrent plus d'origine à l'emploi festif des armes. Pour Castellane, par manque d'enquête apte à révéler des ajustements dans la mise en scène des "huguenots", 9 je m'arrêterai à une hypothèse. Le traitement festif de l'altérité religieuse doit aller régulièrement dans le même sens, que les oppositions campées aient un caractère intra ou interreligieux. Une observation directe mettrait sans doute plus généralement en évidence que l'usage des armes se maintient toujours en contrant l'a priori de l' "ennemi héréditaire". Pour ce qui est sûr, les exemples de Riez et de Breil convergent en ce que les figurants sarrasins se sont dissous, soit tout à fait, soit comme ennemis.

Au-delà de cette différence formelle majeure avec l'Espagne, une convergence est remarquable. De même que dans la région d'Alicante, les "Maures" sont ressortis à l'enquête comme des personnages somme toute positifs, de même, à Breil, ils emportent la sympathie générale et, selon une pseudo-généalogie largement attestée en Provence-Alpes-Côte d'Azur, beaucoup d' "originaires" se perçoivent comme des descendants de Sarrasins. ${ }^{10}$ Dans les deux situations, la mise en représentation de soi à travers la fête exprime la possibilité d'une coexistence religieuse paisible. Le renversement d'une interprétation stéréotypée qui ferait d"“autres" religieux des ennemis obligés constitue de la sorte une première forme d'intervention sur les apparences belliqueuses.

9 Je n'ai pas pu éclaircir ce cas, la "bravade" de Castellane interférant avec le calendrier varois. Des images diffusées par Internet ne sont guère informatives que d'allures générales, de même que la présentation d'Evelyne Duret (1981).

10 Sentiment qui ne rend pas moins distantes les relations quotidiennes avec une population harkie, assez importante depuis 1962 (Lebaudy 1998). 


\section{“BRAVADE”, GENRE ET CHASSE"}

Avant de repérer d'autres ressources que l'évacuation d'adversaires religieux potentiels ou le retraçage de silhouettes symboliques, il convient d'approcher le fait "bravade" à partir d'un trait fondateur, autrement dit des aspects de la virilité qu'il met à l’œuvre.

\section{Du côté des femmes}

Ce n'est pas que les femmes soient absentes des manifestations, mais les formes de leur présence les situent globalement à la marge du maniement des armes. Autrefois, à Barjols, dont la fête célèbre saint Marcel évêque de Die, certaines - des prénommées "Marcelle" - tiraient un coup de feu de leur fenêtre; ce geste ne les assimilait pourtant pas aux "bravadeurs" du cortège. A Saint-Tropez, des épouses ont touché à la poudre, mais il s'agissait de fournir leur mari au gré de ses besoins. Aujourd'hui (enquête en 1998), à côté du président des Bravades ou cepoun, il y existe une "cépoune"12 aux fonctions créées sur mesure car c'est sa compagne ; en haute Provence ou à Signes, des "prieuresses" ont de même existé en retrait du "capitaine" régisseur de "bravade". Les "aubades" (séquences musicales avec tirs au domicile du destinataire) s'organisent en une tournée de l'honneur masculine; les femmes qui y apparaissent sont encore une fois définies par leur statut conjugal, plus rarement filial, ou bien elles sont absorbées par leur métier quand l' “aubade" à un service public neutralise le genre des fonctionnaires. Même ce qui peut paraître une exception confirme cet effacement structurel. Quand par exemple un important renouvellement des costumes festifs vaut une "aubade" à des femmes (Barjols, 2000), son exécution dépersonnalise chacune des couturières puisque l'hommage est adressé en plein air; il ne s'agit aucunement - comme c'est la façon habituelle de procéder - de se rendre à chaque domicile ou au lieu de travail. Par ailleurs, si les femmes sont admises à tirer en fin de fête devant l'effigie qui représente le saint célébré - on dit "faire les honneurs au saint" -, qu'en est-il concrètement? A Barjols, intervenant l'une après l'autre, elles sont rares, jeunes et parmi les plus ferventes de saint Marcel, et des approbations souriantes se font entendre. A Saint-Tropez, où ces tirs sont pratiqués à plusieurs en même temps selon la disponibilité d'armes plus nombreuses, où donc

11 Cette section correspond peu ou prou au sujet que j'avais inscrit au programme du colloque - sans actes - "Fêtes et réjouissances dans l'Europe Méditerranéenne, XVI ${ }^{\mathrm{e}}$-XX $\mathrm{C}^{\mathrm{e}}$ siècle" (Université de Montpellier, 2002). Un tel intérêt pour le genre formait à cette époque (sans que cela me fût connu) un sujet de thèse à partir d'une fête de Maures et chrétiens relativement récente. Une étude (Heuzé 1999) montre ainsi que l'innovation d'une présence féminine y est passée par un temps de travestissement (1962 sq.) puis par une féminisation de l'apparence (à partir de 1975), cependant toujours subordonnée à la figure du "sauvage". L'orientation du propos exclut en conséquence une interrogation sur l'image guerrière semblable à celle qui m'occupe.

12 Cepoun est du provençal, “cépoune" une transposition en français. 
davantage de femmes y accèdent en étant moins individualisées, elles sont en contrepartie plus dépendantes (infra) pour le maniement de celle qui leur est prêtée. Enfin, les quelques cantinières - figures militaires sans armes - qui ont place dans la Bravade de Saint-Tropez n'atteignent qu'environ 1\% de l'effectif; encore ce rôle ne leur est-il accordé que si un homme de leur famille est "bravadeur". Et, à Castellane, si une jeune fille est pleine actrice dans la Fête du pétardier en 1978, elle y est vêtue en homme.

Le plein rôle des femmes réside ailleurs que dans la manipulation de la poudre. A Saint-Tropez, où n'est pas "bravadeur" qui veut, l'ascendance locale de laquelle elles participent peut aider leurs fils à le devenir. Des fonctions féminines classiques sont très sensibles au moment des "aubades", cela dans l'agencement de décors domestiques autour de bustes du saint en réduction; dans la préparation de buffets à base d'aliments aux couleurs rouges et blanches de la localité (saucisson, radis, fraises à la Chantilly...) ; dans le soin pris pour une toilette qui combine volontiers des vêtements de ces deux teintes, de sorte que, lorsque les épouses se tiendront auprès de leur mari "bravadeur", elles l'épauleront de leur propre allégeance tropézienne. La fête s'étendant sur trois jours, le tour le plus apparent des femmes ou plutôt celui des familles sera en fait le dernier grâce à une excursion et un repas champêtres dont la date d'apparition ne m'est pas connue. Avec sa caractéristique bucolique et détendue, cette journée apparaît comme un décentrement géographique et thématique. Au mois de mai où se situe la Bravade, cette séquence opère un glissement, du vacarme des armes vers le délassement collectif fréquent (Bromberger 2008) des collations printanières en plein air.

Au fond, à travers le détail, se dessine une dominante sexuée de divers lieux festifs : masculinité des places ou des voies publiques où retentit la poudre ainsi que de l'Oustau (en provençal "maison", ici siège associatif) de la Bravade, point de rencontre à divers moments de la fête et rappel peut-être, toutes proportions gardées, des "chambrées" ou "chambrettes", qualifiées par Lucienne Roubin (1970a ou, sous forme d'extrait accessible en ligne, 1970b) de "maisons des hommes"; mixité organisée de la procession, ouverte par des femmes et des hommes en vêtement local, et continuée par les "bravadeurs" se succédant par corps en uniformes militaires (mixité au contraire totale de la salle dévolue aux discours et au vermouth d'honneur, ou de la "colline" du piquenique); féminité affichée de l'espace domestique, manifestant en bordure des rues l'engagement personnel de la maîtresse de maison. L'apaisement ne vient pas comme à Breil d'une alliance plutôt inattendue entre "peuple" local et "Sarrasins" mais d'une succession de séquences qui, cumulant des oppositions - hommes/familles, centre urbain/campagne, poudre et évolutions militaires/commensalité et détente -, ne font plus de la Bravade une unique affaire d'armes et de parade pseudo-militaire. Inscrits dans une série de contrastes, les épisodes de tirs sont coupés par des temps de répit. 


\section{Des hommes entre chasse et "bravade"}

Affaire de genre, le plein maniement de la poudre renvoie aussi, on pourrait dire "par conséquent", à la chasse. Si les "bravades" ont une longue histoire c'est en effet en raison de la large et précoce diffusion des armes à feu; dans la Provence d'avant la Révolution déjà, elles sont attestées à tous les échelons sociaux en liaison avec une pratique de la chasse bien ancrée. Des générations durant, l'usage festif de la poudre a reposé sur une activité cynégétique généralisée et, au présent, l'appartenance à la société locale de chasse est quasi automatique, la réglementation exigeant le permis de chasse pour participer à une "bravade".

Accéder à son premier fusil de chasseur ou à son tromblon festif sont, pour les jeunes Provençaux intéressés, des étapes assez semblables sur le chemin qui conduit vers une pratique cynégétique ou festive adulte. De même que le parcours d'un chasseur commence par le piégeage avant qu'un âge suffisant lui acquière l'usage des cartouches, de même, un petit Barjolais par exemple figurera dans la fête "en Provençal " ou en marmiton tout en rêvant des pétards interdits ou en se les accordant furtivement; puis il s'acheminera vers une image plus batailleuse en revêtant une cotte de maille et un casque, et en portant une hallebarde; enfin, il pourra passer dans le groupe des jeunes gens en armes à feu. Les liens entre les domaines festifs et cynégétiques sont si réels qu'un acteur à qui je demandai un jour s'il avait eu la charge d'un tromblon me répondit négativement en s'expliquant par son unique et désastreuse chasse au fusil.

Poursuivant la comparaison, on pourrait noter que les métaphores érotiques que des chercheurs ont mises en évidence avec les fusils de chasse (Bromberger et al. 1980, 1981) parcourent aussi les "bravades". Revoyons la scène de tir devant l'effigie de saint Tropez. Plusieurs personnes s'y adonnant en même temps, il existe une indéniable contrainte de sécurité; toujours est-il qu'une femme peut y être rendue presque complètement passive par un "bravadeur" qui se tient derrière elle pour contrôler l'arme, ce qui l'amène à passer les deux bras autour de sa protégée. Ce moment est bien sûr fugitif, encore que rien n'interdit de renouveler l'expérience, mais il laisse la possibilité d'un rapprochement entre personnes de sexe opposé et notamment entre jeunes personnes. Les réactions suscitées à la terrasse d'un café par une femme qui, répondant à une question à ce sujet, disait avoir “tiré un coup avec X” m'ont d'ailleurs semblé révélatrices, que cette formule malencontreuse ait entraîné, dans le petit groupe attablé, des rires en coin ou des marques de confusion.

Le nouveau porteur d'arme, en amont de son changement d'emploi dans le cortège, aura par ailleurs acquis la capacité à bien supporter l'alcool. C'est là une qualité si nécessaire à un homme qu'elle a été une condition d'entrée dans des "chambrettes". De nos jours (Dufour 1989), la "tournée" entre habitués d'un café reste un élément de base de la sociabilité masculine, soumis à l'aptitude à absorber sans dommage plusieurs verres de pastis [suite page 66] 


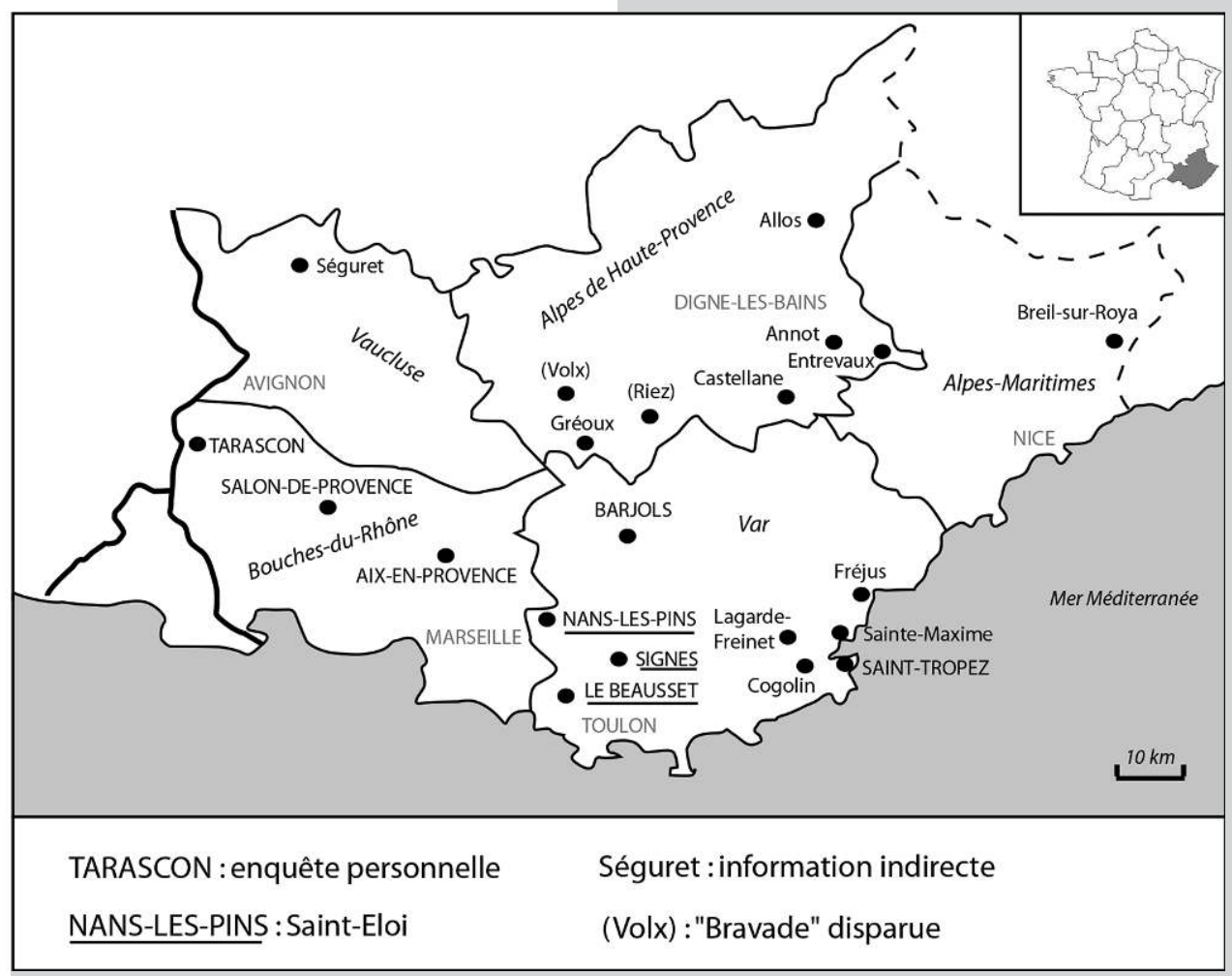

Figure 1 - Lieux cités. (Carte: Gilles Durrenmath, LASMIC) 

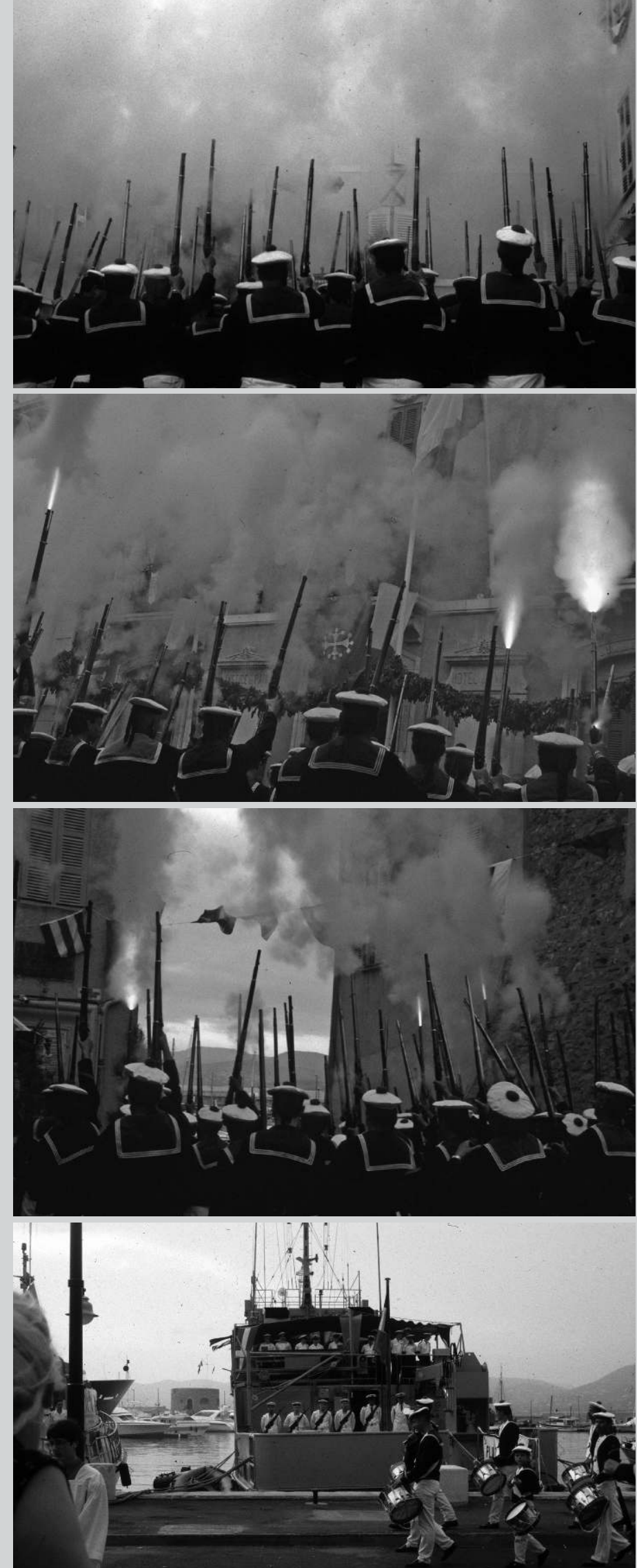

Figure 2

Une allure de guerre:

juste après le tir simultané de "marins". La fumée obscurcit encore un après-midi ensoleillé. Saint-Tropez, Bravade de mai 1998.

(Clichés: D. Dossetto)
Figure 3

Sur le quai de Saint-Tropez, des "bravadeurs" et d'authentiques militaires. Saint-Tropez (Var), Bravade de mai 1998. (Cliché: D. Dossetto) 
Figure 4

Variété des situations et donne vestimentaire: une allure historique et des tromblons. Barjols, Saint-Marcel, 2000.

(Clichés: D. Dossetto)

$4 a$ - Prise des armes: en face du maire, le "capitaine de ville"; en ligne au loin, le "capitaine de troupe" en blanc et à sa droite l' "enseigne" et son "major".

Le concepteur de la séquence

(1954, d'après le modèle de Saint-Tropez) a encore porté un uniforme militaire quand il a été

"capitaine de ville" (1978) La "médiévalisation" des apparences est venue du changement de génération à la tête de l'association organisatrice.

$4 b$ - "Aubade" au cimetière. Se bouchant les oreilles, un membre de l'association organisatrice.

Figure 5

Une déambulation qui occupe l'espace urbain, un petit "tambour-major" en tête. Saint-Tropez, Bravade de mai 1998. (Cliché: D. Dossetto)

Figure 6

L' "enseigne" manipulant le drapeau devant la mairie. Saint-Tropez, Bravade de mai 1998. (Cliché: D. Dossetto)
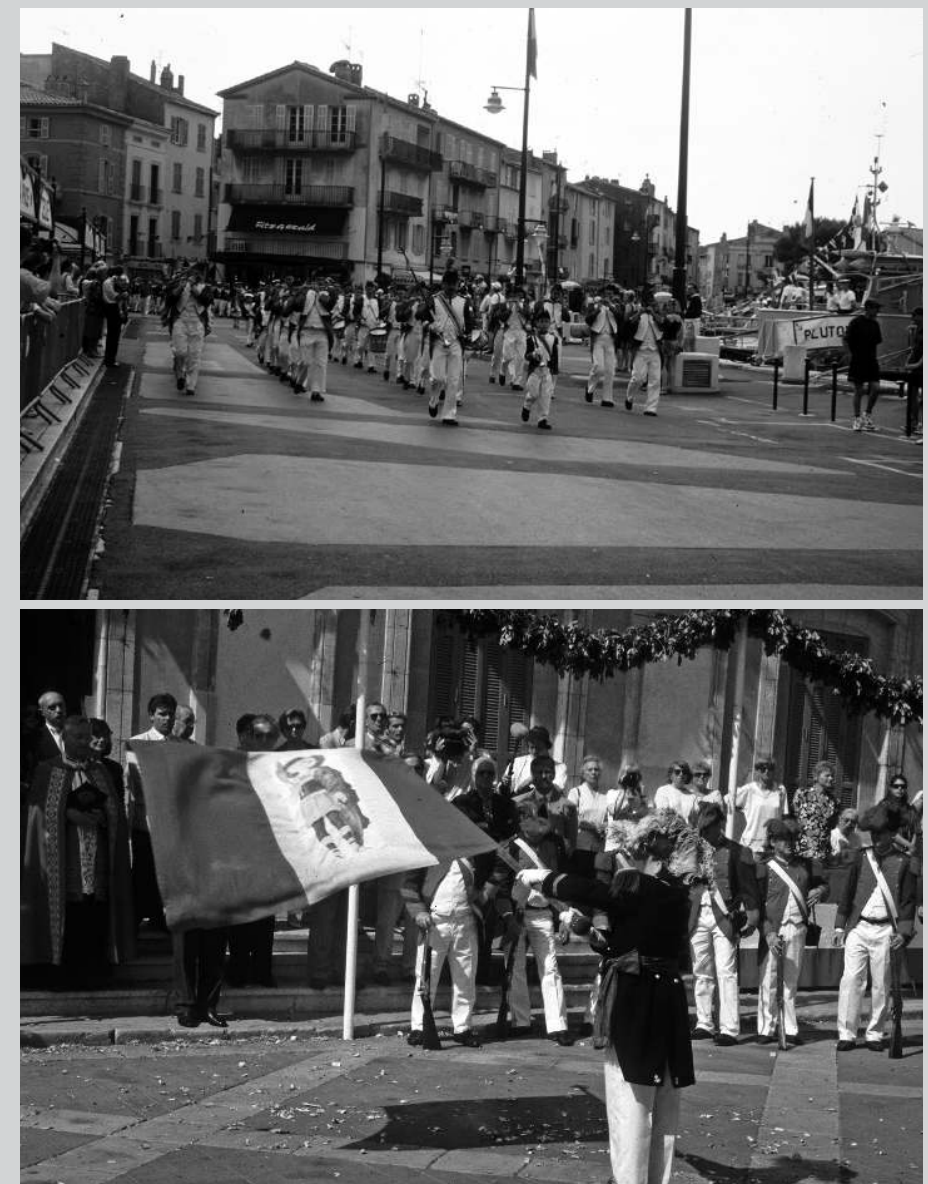


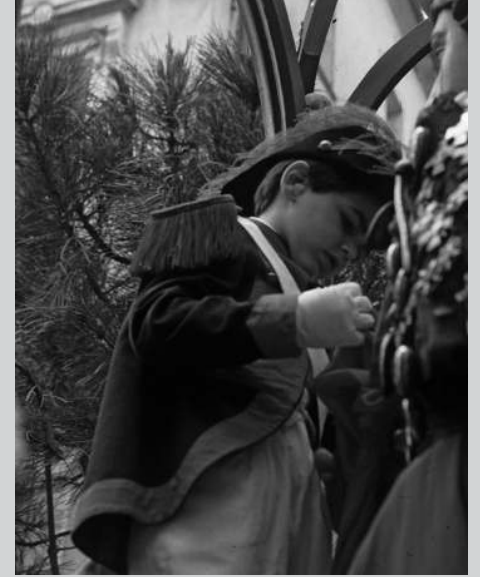

Figure 7

Un rôle prévu pour un petit enfant, hissé à la hauteur du buste patronal. Saint-Tropez, Bravade de mai 1998. (Clichés : D. Dossetto)

$7 a$ - Mise en place d'une écharpe.

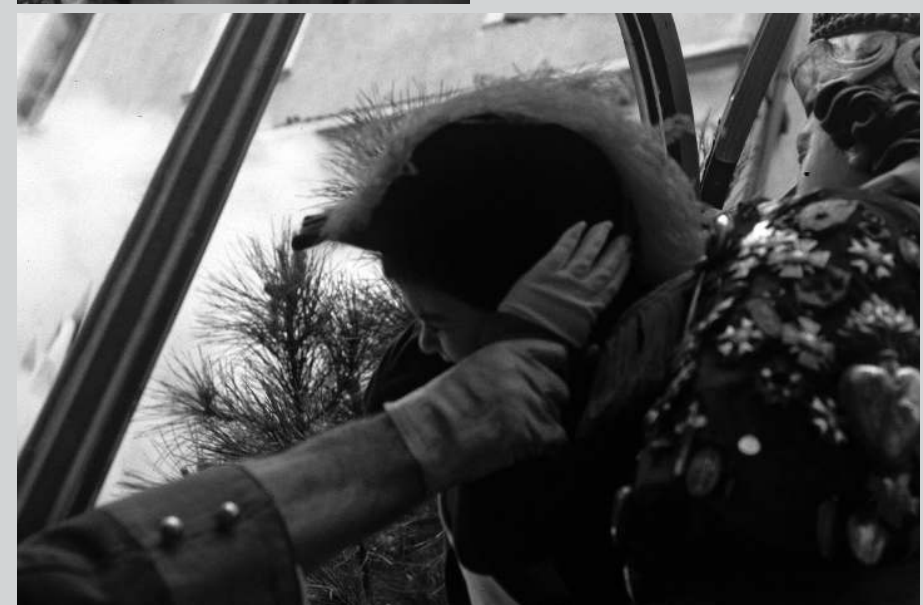

$7 b$ - Secours adulte quand éclate la rafale succédant au baiser.

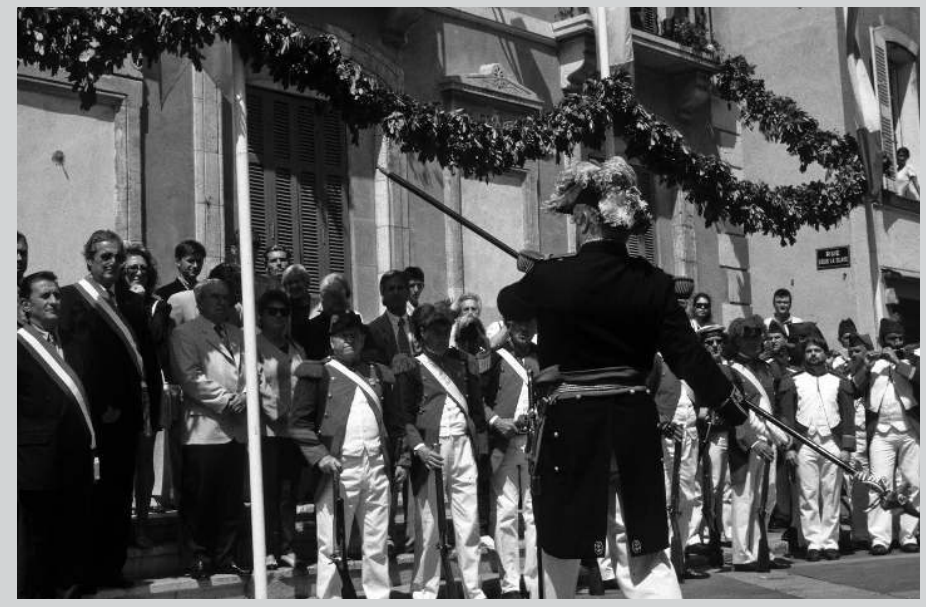

Figure 8

Deux visages du "capitaine de ville”. Saint-Tropez (Var), Bravades de mai 1998 et mai 1954.

(Clichés: D. Dossetto et Louis Buyret)

$8 a$ - Le "capitaine de ville" proprement dit, maniant la pique qui va déclencher une mitraille.

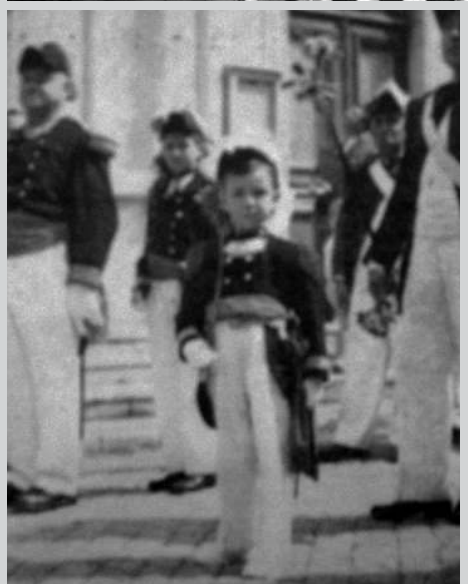

$8 b$ - Un double enfantin il y a quelques décennies déjà. 
[suite de la page 61] (alcool anisé détendu à l'eau). Dans l'exercice festif, le savoir boire permettra de garder la totale maîtrise de son arme malgré les nombreux "pastis" servis au cours d'“ aubades" s'enchaînant parfois toute une journée. Un "bravadeur" ou un autre se plaira sans doute à faire sursauter le public par un tir isolé ou chargera de temps en temps son arme avec une application ostensible et menaçante; des tirs dans les bassins de fontaine ou sur le mur délabré d'un bâtiment ancien pour faire tomber encore un peu de crépi signalent plus nettement des "aubades" bien avancées; mais ce sont là des façons joyeuses et sans risque, autorisées par l'ambiance festive.

Homme d'un statut particulier, le curé peut être plaisanté. Des "bravadeurs" rient de son jus de fruit qui les rendrait malades (il en offre, au moment de l' "aubade", à la place de pastis) et certains s'amusent à garantir leur arme de l'eau bénite dont il asperge leur groupe comme si elle risquait de mouiller la poudre. Un gentil chahut intervient le cas échéant. Au moment des "saluts au saint", assuré qu'il ne s'en rendra pas compte, un "bravadeur" tend par exemple au curé une arme conservant sa baguette, son but étant de l'arrêter avant qu'il ne tire c'est-à-dire ne commette cette erreur de débutant de l'expulser au loin et de la perdre; le prêtre évidemment rit de la farce qui démontre son incompétence.

Devenir "bravadeur" (en armes) - ou "bravadier" - signe au total l'âge viril et ses performances. Selon les lieux, les hommes resteront plus ou moins longtemps actifs. A Saint-Tropez, où l'attachement à la Bravade est très profond, on voit des vieillards ne pas se lasser d'y participer, et beaucoup souhaitent être inhumés dans leur uniforme de "bravadeur". Dans d'autres localités, la pratique des tirs festifs est plutôt une affaire de jeunes gens et il est notable à ce sujet qu'un récent dictionnaire de la France rurale (Lachiver 1997) associe la "bravade" à une catégorie d'âge. ${ }^{13}$ De manière courante toutefois, les pratiques distinguent hommes mariés et célibataires; à Signes par exemple, le nombre des uns et des autres semble désormais fixé à douze.

Certains des aspects qui viennent d'être évoqués ont leur pendant dans les fêtes de Maures et chrétiens, où le goût juvénile pour l'alcool (Albert-Llorca 1995: 10) et les armes à feu (Albert-Llorca 2004: 46) est aussi récurrent. Laissons pourtant en suspens la question de l'âge afin de nous arrêter sur une donnée plus fondamentale du point de vue que nous avons adopté. Chasseurs de fait dans la vie ordinaire, les hommes en armes cultivent parfois cette apparence dans la fête.

L'utilisation d'une arme de chasse dans une "bravade" répète une habitude ancienne, mais elle est ambivalente. Portons-nous par exemple à Signes. Les "bravadiers" y utilisent aujourd'hui le fusil avec lequel ils chassent. Or ils ont

13 Inversement, la "bravade " et sa jeunesse en armes échappent à un échantillon festif international récemment produit par le Centre alpin et rhodanien d'ethnologie (Isnard et Lazier 2010). Sur le lien historique entre "bravade" et "jeunesse", voir Agulhon (1984 [1966]). 
recouru à des tromblons, attestés par exemple dans les années 1960. Tout en les délaissant pour leur arme courante, ils ont conservé redingote et chapeau hautde-forme qu'ils portaient alors. Si l'on considère de plus que la poudre risque d'endommager les fusils modernes, ${ }^{14}$ il ressort qu'au contraire de ce qu'on aurait pu croire à première vue, la facilité ne détermine pas l'équipement actuel.

Linventaire des localités utilisant encore ou à nouveau le fusil de chasse reste à faire, néanmoins ce dernier est assez caractéristique des Saint-Eloi. Par ailleurs, des formules plus originales existent. Le "bravadeur" qui, à Salon, figure dans la fête de la transhumance aux côtés de personnes en costume local est lui-même vêtu comme un groupe folklorique représenterait un chasseur. A Tarascon, la "bravade" est réhabilitée autour du personnage de Tartarin, type littéraire et héros de roman (1872), incompétent cynégétique notoire mais chasseur prétendu de lions; dans la fête, en fusil comme il se doit, il est entouré de quelques traqueurs de fauves en casque colonial, eux aussi dûment équipés. Régulièrement, un jeu de correspondances entre la chasse et la "bravade" permet de moduler l'usage festif de la poudre, et certains enfants confirment le parallèle à leur façon quand, observant l'orientation au ciel des fusils, ils s'inquiètent de ces "bravadeurs" ("bravadiers") cherchant à "tuer les oiseaux".

Le fait que, selon les localités, les acteurs festifs sont propriétaires ou non de leur arme peut évidemment avoir son incidence. Pourtant, si nette que soit la coïncidence avec un certain passé, il apparaît que l'usage même du fusil de chasse ne relève pas d'un comportement routinier ou à moindre coût, mais, au moins dans certains cas, d'une option authentique qu'il faudrait étudier sur quelques décennies. A cette échelle, l'alternance éventuelle des armes amène à penser que les connotations de chacune pourraient plus ou moins compter au nombre des facteurs de changement. Ainsi, le recul, comme à Signes, du tromblon au fusil a-t-il produit un déplacement vers l'évocation d'un loisir ordinaire, au point qu'il est possible de se demander si, succédant à l'emploi d'armes spécifiques, la référence actuelle à la chasse ne tirerait pas la "bravade" hors d'elle-même en brouillant la donne. La question est justifiée, mais l'effet de cette permutation d'armes ne doit pas être exagéré. Il n'est pas assimilable à celui du pique-nique familial tropézien. Ce dernier produit une rupture évidente dans les pratiques (renouvellement du programme, fête annexe emboîtée dans la fête principale). Il en va tout autrement avec une "bravade" en cours de Saint-Eloi. Cette fête se présentant avant tout comme une concentration de chevaux de trait - certains secteurs géographiques la pratiquent d'ailleurs sans "bravade" -, le voisinage animal happerait tout type d'armes dans une sphère plus agreste que guerrière. Un fusil de chasse est certes plus anodin qu'un tromblon, mais il s'inscrit aussi dans une continuité presque logique avec la rusticité de la fête. En somme, la nature de l'arme perd de son 
importance au profit du contexte ou, pour dire autrement les choses - et cette observation est extensible à mes autres exemples -, une modification, ici le passage du tromblon au fusil, n'est évidemment pas univoque. Plus que sur de pseudo-chasseurs, notre attention est en conséquence appelée à se centrer sur des porteurs d'armes à l'allure de miliciens.

\section{CÉLÉBRER UNE FÊTE AUX ALLURES MILITAIRES}

Sans poursuivre la revue des cas de figure, il est clair désormais que les réactivations ou autres seuils festifs peuvent s'accompagner de modifications diverses, calmant métaphoriquement le bruit des armes ou poliçant les combats. En reprenant autrement notre interrogation de départ, il s'agit de s'intéresser maintenant à des moments particuliers. Qu'en est-il des fêtes en armes à des dates où une guerre authentique mobilise les acteurs ou renvoie à leur expérience immédiate?

\section{“Bravade(s)" et guerres effectives}

Les fêtes de Maures et chrétiens ont pu connaître un regain ou une inflexion dans des périodes d'exaltation du sentiment national ; ce fut le cas après l'épopée napoléonienne (Carrasco 1976: 112), sous le régime franquiste ou après la guerre civile, quand, loin d'être célébrée avec des combats, la fête a comporté un "acte de réconciliation" (Albert-Llorca 1995: 7, 2004: 42 et 46). En Provence-Alpes-Côte d'Azur, la guerre de 1914-1918 asséna un coup qui semblait devoir être fatal aux fêtes armées, même là où, juste auparavant, la "bravade" pouvait être conduite par un "vétéran de 1870" (Durbec 1952: 261 et 254). "Nous avons assez longtemps été soldats de vrai, pour ne pas nous déguiser en semble-militaires", résume un érudit (Joannon-Provence 1942: ix, soulignement de l'auteur).

Pourtant, à y regarder de plus près, le dégoût des armes est antérieur. D'une part, des "bravades" associées à des processions avaient auparavant souffert, de manière indirecte, d'une vague d'anticléricalisme. Des décisions municipales interdisant les démonstrations religieuses dans l'espace public - comme, en 1903, à Gréoux dans les anciennes Basses-Alpes (Bertrand 1992) - ont parfois empêché une démonstration de poudre (processionnelle) impossible à huis-clos. Dans ce cas, la guerre a plutôt détourné d'une réactivation qu'elle n'a décidé d'une disparition. D'autre part, la pratique s'essoufflait certainement d'elle-même. Ainsi, à Fréjus (Var), la "bravade" disparut-elle en 1906 à cause d'un accident qui paraît n'avoir été qu'un prétexte (Février 1966: 180) et l'exemple de Saint-Tropez est encore plus net. Vingt hussards participant à la Bravade en 1899 s'évaporent en 1903 et la restauration d'un petit corps d'artilleurs, aussi attesté en 1899, se solde au même moment par un échec. Quant à la liste des "capitaines de ville" qui sont à la tête de la mitraille, 
elle montre qu'à la charnière du XIX ${ }^{e}$ et du XX $X^{e}$ siècles peu d'hommes restent intéressés. Les mêmes individus réapparaissent régulièrement, l'un en 1885 , 1896, 1902 et 1914, l'autre en 1899, 1905 et 1921 . Avec néanmoins ici une autre dimension que celle, habituelle, de repère temporel, la "grande guerre" ne fit que parachever un déclin déjà installé, même si quelques manifestations se maintinrent jusqu'au repère équivalent formé par le conflit de 1939-1945 (Durbec 1952: 260).

Au moins un temps, le phénomène "bravade" a souffert de sa proximité formelle et conjoncturelle avec une guerre. Dans ce contexte, la revivification globalement réussie à Saint-Tropez au cours des années 1930 résulta de la rare conjonction d'une multiplicité de facteurs. Quant aux aspects techniques, il faut signaler la constitution d'une association (1921), l'intervention d'un mainteneur de traditions extérieur à la localité, homme hors du commun par le foisonnement et l'efficacité de son activité (Dossetto 2004b), et le recours à des participants d'autres lieux pour l'accompagnement musical ; une organisation nouvelle et le changement d'échelle dans le recrutement des forces vives fournirent au total les moyens de la réactivation festive. En amont, cette réactivation précoce relevait presque du défaut de choix. Une grave crise économique, le manque d'animations attractives, le fait au contraire que des localités voisines jouaient avec bonheur la carte des fêtes touristiques, bref le besoin d'attirer des visiteurs avaient orienté vers la Bravade les réflexions et les projets de décideurs locaux de toutes tendances idéologiques. Dans l'inventaire des possibles, elle s'était imposée comme ressource locale unique (Girault 1994).

Il n'en reste pas moins que le regain tropézien tranche moins qu'on ne pourrait le penser sur la désaffection générale des "bravades" dans le premier tiers du XXe siècle. Que s'y passe-t-il en effet? La liste des "capitaines de ville" est interrompue durant la première guerre mondiale et, si elle est continue sous la seconde, la Bravade est célébrée "bien entendu" (l'expression est de Buyret 1954) sans armes. Après quoi, en 1945, la pseudo-identité “bravade"/guerre est exploitée pour clore symboliquement le conflit qui vient de s'achever et qui a fait de la localité un centre de Résistance et un "port de la Libération". En pleine Bravade, juste après la messe habituelle "des mousquetaires", l'évêque procède à la "purification" (sic) de l'hôtel ayant abrité la Kommandantur. Des sommités militaires ou politiques sont présentes, qu'il s'agisse d'un ministre et d'un maréchal ayant combattu sur les lieux ou de hauts représentants de la marine nationale et des pays alliés actifs dans la Libération (Ben 1990 [1955]). De plus, les "bravadeurs" ont inscrit dans leur coutumier une nouvelle cérémonie ; remarquablement exempte de poudre, cette commémoration des événements d'août 1944 se présente comme une "bravade" paradoxale.

Soit que d'authentiques guerres nuisent aux fêtes de type "bravade", soit qu'elles leur confèrent une dimension exceptionnelle ou décident d'une célébration supplémentaire dans le calendrier associatif, c'est de toute façon la 
force de l'analogie qui fédère des orientations en apparence contradictoires; il faudra en envisager des conséquences après avoir évoqué l'intérêt occasionnel de cette même analogie.

\section{"Faire parler la poudre"}

L'image du conflit qui adhère aux fêtes armées est susceptible de leur donner une dimension spéciale dans des situations de toute sorte. Dans la région d'Alicante, des rivalités de clocher trouvent dans les modalités festives une expression possible; une ville déchue de son ancien prestige y reprend par exemple un pouvoir symbolique de quelques jours sur une rivale qui s'est développée à ses dépens (Albert-Llorca et Albert 1995). A une autre échelle, la présentation festive des Maures exprimant la possibilité de coexistence religieuse désigne implicitement pour ennemi de la population locale le pouvoir castillan (Albert-Llorca 2004). En ce qui concerne l'ancienne Provence, Albert Giraud (1995) réunit maints exemples anciens qui mêlent pratiques festives, positions idéologiques, émeutes, etc. Les conflits entre cléricaux et anticléricaux à la charnière des XIX ${ }^{e}$ et XXe siècles fournissent le cadre d'une revivification éphémère de la Bravade à Saint-Tropez; l'étude détaillée en a été faite (Girault 1994). En raison de l'acuité de leur souvenir, de telles tensions marquent encore, en 1953, la restauration, sous forme de "reconstitution historique", de la "bravade" pour la Saint-Sébastien à Gréoux; la parade est bien "la réoccupation de la voie publique par les solennités confessionnelles" (Bertrand 1992: 435). Au présent, Saint-Tropez fournit une des plus vives utilisations symboliques des armes.

Depuis la fin du XIX ${ }^{\mathrm{e}}$ siècle, peintres, tourisme, cinéma, néo-résidents fortunés ont distingué le village en renouvelant en même temps ses dimensions. Il suffit de feuilleter au hasard un numéro de la Revue du Golfe cö̈ncidant avec une Bravade pour en trouver une présentation bilingue (français et anglais) voisinant avec une information sur des célébrités et les luxueux établissements qu'elles fréquentent. Dans un contexte qui a même entamé le nom de la localité ("Saint-Trop'”) et alors que la population autochtone est devenue minoritaire, l'enracinement local, au moins chez certains, s'est transformé en une valeur exacerbée, réalité décelable à des indices comme un conservatisme inattendu de la langue vernaculaire, noté il y a une trentaine d'années par les dialectologues de l'Atlas linguistique et ethnographique de la Provence. D'une certaine façon, la Bravade aussi est langage. Elle apparaît comme une façon de manifester son appartenance à la localité historique et de reprendre symboliquement possession de la cité. Le maire confie l'autorité sur les lieux au “capitaine de ville” et la ville dote celui-ci d'une "épée en argent” (Fabbri 1990). Être admis comme "bravadeur" et, au sommet de la hiérarchie des hommes armés, comme "garde-saint" est très convoité. Durant la fête, beaucoup de Tropéziens aiment à pavoiser de rouge et de blanc leurs façades etc. Alors que 
le fort impact sonore des tirs indispose vite ceux qui n'y sont pas préparés, ${ }^{15}$ le parcours de l'espace urbain en "faisant parler la poudre" revient à peu près à "se faire entendre" comme héritiers du Saint-Tropez historique.

Que les fêtes armées se présentent éventuellement comme une ressource expressive ne lève cependant pas les aspects, pour ainsi dire trop prononcés de nos jours, d'une allure guerrière ; c'est l'aménagement de ce point délicat qu'il convient d'examiner maintenant.

\section{Adoucir des "troupes" festives}

L'analogie entre guerre et "bravade" est particulièrement sensible quand les hommes en armes se produisent en uniformes comme à Saint-Tropez. Là, la fête met en présence, aujourd'hui comme en 1954, de vrais militaires qui sont "salués" par les "bravadeurs", soit devant la mairie, soit de manière plus caractéristique sur le port. Bien que de tels "saluts" honorent d'autres personnalités comme les élus locaux, en face de représentants d'une authentique armée, il s'instaure une continuité d'autant plus remarquable qu'il y a réciprocité d'action (Buyret 1954: 29; Fabbri 1990). Une donnée primordiale est donc que les uniformes des "bravadeurs" sont assez anciens - avec bicorne et autres - pour ne pas court-circuiter les souvenirs familiaux ou une expérience personnelle; le mélange de silhouettes relevant d'époques différentes est d'ailleurs habituel aux "bravades" figurant des régiments de soldats. Tel qu'il s'est opéré à Barjols, le remplacement des uniformes signalant l'“ état major" par des costumes médiévaux creuse encore davantage l'écart entre la guerre connue ou proche et la "bravade".

En plus de la distanciation par le renvoi vestimentaire à un passé opportunément lointain, il faut noter comme deuxième trait général une tendance au rajeunissement des troupes. Il peut être total comme à Fréjus quand, en 1952, des enfants furent requis d'endosser des uniformes antérieurement portés par des hommes (Février 1966) ; mais le cas est sans doute rare car une telle formule suppose de renoncer à l'emploi essentiel de la poudre. Ce sont donc les "états-majors" (toutes les manifestations de type "bravade" n'en n'ont pas) qui, dotés d'insignes de commandement autres que des armes à feu - pique, armes blanches, drapeau -, se ressentent surtout de cette évolution. Le même mouvement s'observe à Barjols et à Saint-Tropez où il est plus poussé. Pour le comprendre, il faut savoir que les dignitaires des "bravades" représentent sur la durée, les uns les hommes mariés, les autres la "jeunesse” (célibataire). Malgré les connaissances historiques des organisateurs de "bravades" à ce sujet, il s'opère un glissement de cette jeunesse adulte vers l'adolescence voire la préadolescence, et ce rajeunissement affecte l' "enseigne" et son "major" ou 
“aide de camp". A Saint-Tropez, les tenants de ces fonctions sont carrément imberbes et c'est vrai depuis plusieurs décennies comme le montrent les clichés de 1954; en 1998, leur mère les suit discrètement lors des démonstrations armées pour les soulager aussitôt que possible à force bouteilles d'eau et autres.

Si le rajeunissement de l' "état major" est un trait partagé, d'autres tempéraments, en l'état de ma documentation, semblent propres à Saint-Tropez et, bien que j'ignore leur date d'apparition, ils m'apparaissent tous tardifs (Lally et Condroyer, en 1888, ne les signalent pas). A défaut de connaître la fleur au fusil, la Bravade recourt à de minis bouquets bénits, arborés notamment lors d'une procession pour laquelle les hommes en armes délaissent leur tromblon ou autre pour une épée; cela fait au moins un demi-siècle qu'ils se fleurissent ainsi (Buyret 1954: 29 et 31). Les occasions d'accolades entre eux sont nombreuses et, devant le siège de l'association, ils défilent en plus pour embrasser le cepoun et la "cépoune". Le retour du pique-nique entre aussi dans la liste de ces amendements apaisants; pendant une farandole (danse en chaîne fréquente en clôture d'un banquet) tournant à la course au flanc d'une colline, hommes ou femmes sont saisis par surprise par plusieurs "bravadeurs" unis dans un cercle impromptu, et projetés plusieurs fois en l'air à l'amusement général; n'importe quel porteur d'uniforme peut ainsi se trouver dans une position très peu martiale.

Mais, prolongeant la "puérilisation" de l' "enseigne" et de l' "aide de camp", c'est surtout la présence de tout jeunes "bravadeurs" (quatre-six ans) qui est remarquable. Fait massif aussi en 1954, ils sont nombreux, vêtus comme les adultes, à accompagner les troupes en procession. Trois d'entre eux, sans doute plus nouveaux que les autres, sont davantage individualisés. Un est au centre d'une séquence spécifique; il noue une écharpe au buste de Saint Tropez et "après [...] s'être décoiffé embrasse le saint sur les deux joues" (Fabbri 1990). Moment très attendu aujourd'hui, ce baiser au buste reliquaire n'est évoqué ni dans le compte-rendu ni dans l'iconographie de 1954; on peut raisonnablement en déduire son inexistence à cette date et la création récente de ce rôle enfantin. Un très jeune "tambour-major" imite scrupuleusement son aîné; il n'y a pas d'homologue photographié ou évoqué en 1954. Par son titre et son vêtement, un petit garçon est un double du "capitaine de ville". Un cliché nous montre son prédécesseur dans cette fonction en 1954, mais sans que Louis Buyret s'attarde à un commentaire; la description étant par ailleurs très précise, il faut peut-être voir dans cet intérêt modéré l'indice d'une innovation non encore véritablement assise. Quoi qu'il en soit, ce petit personnage a une utilité. Comme acteur central, le "capitaine de ville", en gagnant, par l'enfant, un second visage, a perdu un peu de son allure terrible. Adulte, "il" ordonne normalement la mitraille du geste lent et grave de sa pique mais, garçonnet, "il" a un rôle épisodique de parade et sa petite taille en fait une figure attendrissante au milieu des hommes en armes. La candeur de l'enfance tend ainsi à ébranler la correspondance entre fête armée et guerre, à blanchir 
en quelque sorte la poudre. Autrement dit, même les "bravades" en uniformes se révèlent souples à la métamorphose pour atténuer leur caractère belliqueux.

\section{TRADITIONNELLE, MAIS EN PORTE-À-FAUX}

Si l'on procède à une récapitulation, ceci finit par s'imposer. Le dossier "Bravade" apporte d'ores et déjà plusieurs exemples de travail sur l'analogie milicienne. Malgré sa longueur, la série des interventions n'est pas close : allures ludiques ou dimension carnavalesque; détente résultant de l'insertion de séquences sans armes, d'un changement de cadre, de moments familiaux collectifs ; transposition de conflits dans le registre social ou évocation cynégétique multiforme; jeu sur le vêtement avec la floraison de costumes "historiques" (Barjols, Castellane, Annot, Entrevaux...) ou plus ou moins "bourgeois" (Signes, Le Beausset, Nans-les-Pins...) pour les hommes en armes, et la contribution de groupes folkloriques en coiffes et autres; adoucissement direct des hommes en soldat (accolades, bouquets bénits) ; infantilisation partielle progressive et valorisation de l'extrême jeunesse. Ces amendements sont plus ou moins frappants selon que la manifestation, dite "Bravade", est d'allure militaire ou qu'elle ressortit au genre "fête à bravade" (cf. Dossetto 2009), dont la composition complexe est moins susceptible d'être traversée par le spectre guerrier. Mais ce sont autant de descripteurs ou de tendances qui ont des équivalents ailleurs. A Villajoyosa, on a souligné le recours au burlesque ou la multiplication de duels se terminant en embrassades après deux coups d'épée (Albert-Llorca 1995: 8). Les "marches" belges méritent aussi un coup de projecteur. Les sapeurs y ont remplacé les tabliers de cuir blanc, partie prenante de leur uniforme, par des tabliers brodés; même si elles ne les confectionnent pas toujours, les mères ou les épouses assurent leur entretien complexe etc.; elles sont entrées de la sorte dans une fête où leurs compagnons de vie se signalent désormais par une toile ajourée à fleurs en rapport esthétique avec le linge de maison (Pop 1986). Une vingtaine d'années après cet état des lieux, des femmes en viennent cependant à contester le tablier brodé comme "symbole de leur ancienne sujétion au marcheur mâle" (Foulon 2009); elles revendiquent du même coup une participation directe aux défilés, auxquels elles apporteraient certainement un autre caractère "euphémisant".

Bien que la liste tirée d'études mues par leurs propres questionnements soit forcément réduite, l'attestation de tempéraments comparables sur des terrains différents, et leur cumul éventuel comme à Saint-Tropez, confirme leur intérêt général et leur finalité. Très sérieux quand on l'aborde du point de vue loco-identitaire, le fracas festif des armes est de plus en plus l'objet d'une élaboration intégrant des formes d'atténuation. Cette actualisation pourtant ne relève pas à proprement parler ou pas seulement du maquillage ou de l'estompe - la consommation de poudre augmente -, mais plutôt de la mise en abyme. 
Elle provoque un effet de flou, comme un "bougé" photographique. C'est un trait fort des fêtes à l'examen, un processus partagé au-delà d'une variété factuelle au contraire cultivée avec soin. Il en va comme si elle était un accommodement nécessaire ou salutaire, une mesure de légitimation que certaines des données réunies inscrivent avec certitude dans une durée relative.

Afin de mieux percevoir le travail spécifique sur la poudre festive, prenons un recul qui permette de le rapprocher de celui qui signale des fêtes malmenant symboliquement un animal. Dans un village de Croatie, le déroulement traditionnel prévoirait un abattage public; à l'orée des années 2000, la pression publique oblige à le remplacer par une imitation évocatrice (CapoZmegac 2004 et information directe). A Barjols et dans sa Saint-Marcel dont nous avons évoqué la "bravade", pendant des décennies, un bœuf a paradé vivant avant d'être exhibé embroché tête intacte; depuis 1995, deux bœufs se succèdent dans ces séquences pour laisser le premier regagner ses "verts pâturages" (sic) et rapprocher le second d'une bête de boucherie ordinaire (Dossetto 2001). Qu'il s'agisse de fêtes armées ou de telles démonstrations festives de la mort animale - on sait par ailleurs combien la corrida mobilise de détracteurs -, l'usage (en cours de réactivation ou établi, peu importe) a également opposé à des mainteneurs de particularismes locaux une violence inhérente dérangeante. Les aménagements trouvés tendent à diminuer la sorte de malaise, la gêne comparable qui en résultaient. Des pratiques en apparence bien différenciées gagnent au total à être envisagées globalement comme des faits de tradition en porte-à-faux, porte-à-faux dynamique puisqu'il génère en quelque sorte un changement qui soit apte à le réduire.

\section{BIBLIOGRAPHIE}

AGULHON, Maurice, 1984 [1966], “Les organisations municipalisées : guet, 'bravade', jeunesse ”, Pénitents et Francs-Maçons de l’ancienne Provence. Paris, Fayard, 43-64.

ALBERT-LLORCA, Marlène, 1995, "Maures et Chrétiens à Villajoyosa : une ville, sa fête, son saint”, Archives des Sciences Sociales des Religions, 91 : 5-19.

ALBERT-LLORCA, Marlène, 2004, “Une histoire à (re)jouer : les fêtes valenciennes de Moros y Cristianos", in J.-L. Bonniol et M. Crivello (dirs.), Façonner le passé: Représentations et cultures de l'histoire (16 $6^{e}-21^{e}$ siècle). Aix-en-Provence, Publications de l'Université de Provence, 37-52.

ALBERT-LLORCA, Marlène, et Dominique BLANC, 2001, "Faut-il brûler anachronisme? Souci historien et déni de l'histoire dans les rites festifs”, in A. Bensa et D. Fabre (dirs.), Une histoire à soi: Figurations du passé et localités. Paris, Maison des sciences de l'homme et Mission du patrimoine ethnologique, 87-102. 
ALBERT-LLORCA, Marlène, et Jean-Pierre ALBERT, 1995, "Mahomet, la Vierge et la frontière", Annales: Histoire, Sciences Sociales, 50 (4) : 855-886.

ALBERT-LlORCA, Marlène, et José Antonio GONZÁLEZ ALCANTUD (dirs.), 2003, Moros y Cristianos: Representaciones del Otro en las Fiestas del Mediterràneo Occidental. Grenade, Diputación de Granada et Toulouse, Presses Universitaires du Mirail.

BEN, Ferdinand Jean, 1990 [1955], Le pays de Saint-Tropez. Saint-Tropez, Amis de la Bravade et des traditions tropéziennes.

BERTRAND, Régis, 1992, "Fête patronale et souvenirs historiques: un ethnotexte sur la ‘bravade' de Gréoux-les-Bains”, Le Monde Alpin et Rhodanien, 1992 (1-4) : 41 1-436.

BROMBERGER, Christian, et al., 1980, "Les paysans varois et leurs collines : les enjeux symboliques d'une 'passion', I ère partie”, Forêt Méditerranéenne, II (2) : 193-200.

BROMBERGER, Christian, et al., 1981, “Les paysans varois et leurs collines : les enjeux sym-

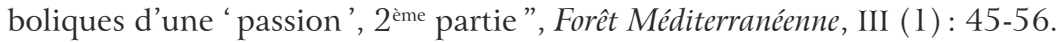

BROMBERGER, Christian, 2008, “De la ribote en Provence au sizdah bedâr au Gilân (Iran) : variations sur deux modes de sociabilité conviviale”, in C. Bromberger et M.-H. Guyonnet (dirs.), De la nature sauvage à la domestication de l'espace: Enquêtes ethnologiques en Provence et ailleurs. Hommage à Annie-Hélène Dufour. Aix-en-Provence, Publications de l'Université de Provence, 47-55.

BUYRET, Louis, 1954, “Les Bravades de St-Tropez, $2^{\text {ème }}$ partie : En arrivant sur les lieux... ”, Folklore, 19: 23-34.

CANESTRIER, 1955, "La hallebarde dans les fêtes villageoises du Comté de Nice”, Arts et Traditions Populaires, $1: 23-32$.

CAPO-ZMEGAC, Jasna, 2004, “Identité nationale et relance de tradition : l'opinion publique croate et le rituel de la kumpanjija de l'île de Korcula”, in D. Dimitrijevic (dir.), Fabrication des traditions, intention de modernité. Paris, Maison des sciences de l'homme, 87-97.

CARENINI, André (dir.), 1996, Le Bacchu-Ber et la danse des épées dans les Alpes occidentales. Aix-en-Provence, Edisud.

CARRASCO, M.-S., 1976, "La Fête de Maures et des Chrétiens en Espagne: histoire, religion et théâtre”, Cultures, 1: 94-122.

DOSSETTO, Danièle, 2001, "Produits carnés, sensibilité animalière et tradition : les bœufs festifs de Barjols (Var)”, Études Rurales, 157-158: 141-158.

DOSSETTO, Danièle, 2004a, "Vers des fêtes de quelle 'provençalité'? Une tendance du renouvellement festif en Provence-Alpes-Côte d'Azur”, Le Monde Alpin et Rhodanien, 2004 (3-4): 123-140.

DOSSETTO, Danièle, 2004b, “Diversité des situations et récurrences, à travers restaurations et revivifications festives: La Saint-Marcel - ou 'Fête des tripettes' - à Barjols (Var) de 1768 à 1950", in C. Bromberger, D. Chevallier et D. Dossetto (dirs.), De la châtaigne au Carnaval: Relances de traditions dans l'Europe contemporaine. Die, A Die, 74-80.

DOSSETTO, Danièle, 2009, “'Bravades' d'aujourd'hui : poudre en fêtes et hommes en circulation ”, Cahier du CEVETH, 2009: 26-34.

DUfOUR, Annie-Hélène, 1989, “Café des hommes en Provence”, Terrain, 13:81-86, et 14 (erratum : conclusion disparue à l'édition principale).

DURBEC, J.-A., 1952, "Notes historiques sur quelques pèlerinages, processions, fêtes et jeux en Provence”, Actes du 77ème congrès des sociétés savantes. Paris, Comité de travaux historiques et scientifiques, 247-286. 
DURET, Évelyne, 1981, Fêtes de Haute Provence: Calendrier illustré et commenté. Marseille, Laffitte.

FABBRI, A., 1990, Coutumier de la Bravade, dactyl., 34 pp.

FÉVRIER, Paul-Albert, 1966, "Fêtes religieuses de l'ancien diocèse de Fréjus", Provence historique, 44 : 163-189.

FOULON, Pierre-Jean, 2009, "Les tabliers brodés dans les marches folkloriques de l'EntreSambre-et-Meuse (Belgique)", in F. Cousin et N. Pellegrin (dirs.), Tabliers au masculin, tabliers au féminin. Chauvigny, Association des publications chauvinoises, 85-93.

GIRAUD, Albert, 1995, "Des bravades qui tournent mal... De la fête à l'émeute en pays varois", in Les soulèvements populaires en Provence: Emeute, révolte, révolution - Actes des sixièmes journées d'histoire de l'espace provençal (Mouans-Sartoux, 1989). Mouans-Sartoux, Centre régional de documentation occitane, 95-105.

GIRAULT, Jacques, 1994, “Socialisme hégémonique et fêtes traditionnelles : l'exemple du Var dans l'entre-deux-guerres”, in A. Corbin, N. Gérôme et D. Tartakowsky (dirs.), Les usages politiques des fêtes aux XIXe-XXe siècles. Paris, Publications de la Sorbonne, 238-248. HEUZÉ, Laure, 1999, “Des femmes ensauvagées: las 'Negras' de la fête de Moros y Cristianos à Petrer”, Clio: Histoire, Femmes et Sociétés, 10, en ligne à <http://clio.revues. org/260> (dernière consultation en février 2014).

ISNART, Cyril, et Isabelle LAZIER (dirs.), 2010, "Figures de la jeunesse: fête, ruralité et groupes de jeunes", numéro spécial de Le Monde Alpin et Rhodanien.

JOANNON dit PROVENCE, Marcel, 1942, Calendrier des fêtes aux pays de Provence. La Valette, École des cadres du Coudon, $2^{\text {ème }}$ édition.

LACHIVER, Marcel, 1997, Dictionnaire du monde rural: Les mots du passé. Paris, Fayard.

LALLY, Julien, et François CONDROYER, 1888, Coutumier ou guide de la Bravade [...] de SaintTropez. Draguignan, Latil.

LEBAUDY, Guillaume, 1998, “A Stacada d'Breï : le sens de la fête”, Viva ar nostr ber Brei! (Vive notre beau Breil!): Une approche ethnologique de la territorialité dans les Alpes du sud. Aix-en-Provence, Université de Provence, mémoire de maîtrise en ethnologie (direction A.-H. Dufour), 118-136, dactyl.

POP, Denise, 1986, “Le tablier des sapeurs”, Ethnologie Française, 2: 177-190.

ROUBIN, Lucienne A., 1970a, Chambrettes des Provençaux: Une maison des hommes en Méditerranée septentrionale. Paris, Plon.

ROUBIN, Lucienne A., 1970b, “Espace masculin, espace féminin en communauté provençale ", Annales : Economies, Sociétés, Civilisations, 2 :537-560, en ligne à < http://www.persee. fr/web/revues/home/prescript/article/ahess_0395-2649_1970_num_25_2_422234> (dernière consultation en février 2014).

SEIGNOLLE, Claude, 1967 [1963], "Les bravades", Le folklore de la Provence. Paris, Maisonneuve et Larose, 219-225.

VOVELLE, Michel, 1976, Les métamorphoses de la fête en Provence de 1750 à 1820. Paris, AubierFlammarion. 